\title{
Nanostructured Barium Titanate/Carbon Nanotubes Incorporated Polyaniline as Synergistic Electromagnetic Wave Absorbers
}

\author{
Lujun Yu, Yaofeng Zhu, Chen Qian, Qiang Fu, Yongzhen Zhao, and Yaqin Fu \\ Key Laboratory of Advanced Textile Materials and Manufacturing Technology, Ministry of Education, \\ Zhejiang Sci-Tech University, Hangzhou 310018, China \\ Correspondence should be addressed to Yaofeng Zhu; yfzhu@zstu.edu.cn
}

Received 6 January 2016; Revised 10 March 2016; Accepted 29 March 2016

Academic Editor: Donglu Shi

Copyright ( 2016 Lujun Yu et al. This is an open access article distributed under the Creative Commons Attribution License, which permits unrestricted use, distribution, and reproduction in any medium, provided the original work is properly cited.

\begin{abstract}
The three-dimensional (3D) conductive network structures formed by barium titanate/carbon nanotubes incorporated polyaniline were favorable for strengthening electromagnetic absorption capability. Herein, an easy and flexible method consisting of sol-gel technique, in situ polymerization, and subsequent mechanical method have been developed to prepare the barium titanate/carbon nanotubes incorporated polyaniline $\left(\mathrm{CNTs} / \mathrm{BaTiO}_{3} / \mathrm{PANI}\right.$ or $\left.\mathrm{CBP}\right)$ ternary composites. The dielectric properties and microwave absorption properties of $\mathrm{CNTs} / \mathrm{BaTiO}_{3} / \mathrm{PANI}$ composites were investigated in the frequency range of $2-18 \mathrm{GHz}$ by vector network analyzer. Interestingly, it is found that the $\mathrm{CNTs} / \mathrm{BaTiO}_{3} / \mathrm{PANI}$ composites with $3 \mathrm{D}$ conductive network structures presented outstanding electromagnetic absorption properties, which may be attributed to the high impedance matching behavior and improved dielectric loss ability and novel synergistic effect. Additionally, it also can be supposed that the "geometrical effect" of composite was more beneficial to absorbing the incident electromagnetic wave. The $\mathrm{CNTs} / \mathrm{BaTiO}_{3} / \mathrm{PANI}$ composite (the mass ratio of $\mathrm{CNTs} / \mathrm{BaTiO}_{3}$ to PANI is $2: 3$ ) exhibits the best microwave absorption properties, of which the minimum reflection loss value can reach $-30.9 \mathrm{~dB}$ at $8 \mathrm{GHz}$ and the absorption bandwidth with a reflection loss blew $-10 \mathrm{~dB}$ ranges from 7.5 to $10.2 \mathrm{GHz}$.
\end{abstract}

\section{Introduction}

Arising from the rapid development of information technology, the military stealth technique has attracted increasing attention owning its application in the modern warfare. Also, electromagnetic interference has greatly threatened human health and disturbed all contemporary electrical and electronic systems from daily life to space exploration $[1,2]$. Thus, it is urgent demand for a high-performance microwave absorbing material with low density, tiny thickness, strong wave absorption, and broad bandwidth [3].

In this context, carbon nanotubes (CNTs) can be a promising material for reduction electromagnetic radiations due to the combined light weight and remarkable mechanical and electronic properties [4-6]. However, the well conductivity of carbon nanotubes used as microwave absorbing material has the shortcoming of poor impedance matching. In this regard, magnetic materials and dielectric materials may be favorable to improving the impedance matching of
CNTs [7]. For example, the reflection loss of PP magnetic polymer nanocomposites with MWNTs is achieved $-20 \mathrm{~dB}$ at $20.0 \mathrm{GHz}$ [2]. Besides, carbon nanotubes combination with a dielectric material also can improve the impedance matching and induce losses like capacitor effect, antenna effect, polarization effect, and so forth. Barium titanate $\left(\mathrm{BaTiO}_{3}\right)$ [8] represents one of the most studied dielectric materials due to its high dielectric constant, positive temperature coefficient, and nonlinear optical properties. For example, Huang et al. [9] synthesized $\mathrm{BaTiO}_{3} / \mathrm{MWCNT}$ nanocomposites with the mass ratio $\mathrm{BaTiO}_{3}$ :MWCNT $=25: 1$ which exhibited excellent absorption properties. Zhu et al. [10] found that MWCNT covered with $\mathrm{BaTiO}_{3}$ possessed better microwave absorbing properties than the pure MWCNTs in the frequency range 11-15 GHz. In addition, conducting polymers have attracted a great deal of attention as microwave absorber owing to their distinct features. Among the different conducting polymers, polyaniline (PANI) has been the material of choice due to 
various reasons including lightweight, corrosion resistance, facile synthesis, good environmental stability, controllable electrical conductivity, and dielectric loss ability [11, 12]. Guo et al. [13] fabricated a PANI/epoxy nanocomposite with high quality dispersion of the PANI in epoxy matrix, and the uniformly dispersed structure endowed nanocomposite with good mechanical property and outstanding electrical conductivity $\left(3.8017 \times 10^{10} \Omega \mathrm{cm}\right)$. Besides, PANI has the potential application in the field of energy storage, energy saving, and anticorrosion [14]. In particular, it also has become an ideal choice as a component of microwave absorber to achieve a perfect impedance matching.

In present work, to attain an impedance match to achieve greater microwave absorption properties, nanostructured $\mathrm{CNTs} / \mathrm{BaTiO}_{3}$ and $\mathrm{PANI}$ have been incorporated together by a facile approach to form a stable, effective, light weight, and environmentally friendly microwave absorber of $\mathrm{CNTs} / \mathrm{BaTiO}_{3} / \mathrm{PANI}$ composite. It is anticipated that the $\mathrm{CNTs} / \mathrm{BaTiO}_{3} / \mathrm{PANI}$ composite shows excellent microwave absorbing properties, owing to high impedance matching, enhanced synergistic effect, and improved dielectric loss. Meanwhile, it is also worth mentioning that the 3D conductive network structures of $\mathrm{CNTs} / \mathrm{BaTiO}_{3} / \mathrm{PANI}$ composite with greater microwave absorption properties may be affected by the "geometrical effect."

\section{Experimental}

2.1. Materials. Aniline (An) and butyl titanate $\left(\mathrm{Ti}\left(\mathrm{OC}_{4} \mathrm{H}_{9}\right)_{4}\right)$ were purchased from Tianjin Kemiou Chemical Reagent Co., Ltd. Barium acetate $\left(\mathrm{Ba}\left(\mathrm{CH}_{3} \mathrm{COO}\right)_{2}\right)$ and other chemicals were purchased from Aladdin Industrial Corporation. Carbon nanotubes (diameter, $40-70 \mathrm{~nm}$ ) were provided by Wako Pure Chemical Industries, Ltd.

2.2. Preparation of $\mathrm{BaTiO}_{3}$ Sol. $1.7 \mathrm{~g}$ of $\mathrm{Ti}\left(\mathrm{OC}_{4} \mathrm{H}_{9}\right)_{4}$ was dissolved in the mixed solution of $1 \mathrm{~mL}$ of $\mathrm{CH}_{3} \mathrm{COOH}$ and $10 \mathrm{~mL}$ of $\mathrm{CH}_{3} \mathrm{CH}_{2} \mathrm{OH}$ at room temperature to form titanium sol. Then $1.275 \mathrm{~g}$ of $\mathrm{Ba}\left(\mathrm{CH}_{3} \mathrm{COO}\right)_{2}$ dissolved with the mixed solution of $1 \mathrm{~mL}$ of distilled water and $4 \mathrm{~mL}$ of $\mathrm{CH}_{3} \mathrm{COOH}$ was added dropwise into the titanium sol. After a reaction at $40^{\circ} \mathrm{C}$ for $2 \mathrm{~h}$ under constant stirring, a homogeneous transparent $\mathrm{BaTiO}_{3}$ sol was formed.

2.3. Synthesis of the $\mathrm{CNTs} / \mathrm{BaTiO}_{3}$ Composites. $0.05 \mathrm{~g}$ of the $\mathrm{CNTs}$ was oxidized by $\mathrm{HNO}_{3}$ and then added into $\mathrm{BaTiO}_{3}$ sol system under sonicated for $1 \mathrm{~h}$. The $\mathrm{CNTs} / \mathrm{BaTiO}_{3}$ xerogel was obtained by the sol system aged for $24 \mathrm{~h}$ at room temperature and then dried for $24 \mathrm{~h}$ at $60^{\circ} \mathrm{C}$. Finally, the $\mathrm{CNTs} / \mathrm{BaTiO}_{3}$ xerogel transferred to a tube furnace and annealed at $700^{\circ} \mathrm{C}$ for $1 \mathrm{~h}$ under nitrogen atmosphere to obtain $\mathrm{CNTs} / \mathrm{BaTiO}_{3}$ (CB).

2.4. Preparation of $\mathrm{CNTs} / \mathrm{BaTiO}_{3} / \mathrm{PANI}$ Composites. $2 \mathrm{mmol}$ of aniline was added into $10 \mathrm{~mL}$ of $\mathrm{HCl}(1 \mathrm{~mol} / \mathrm{L})$ under stirring condition. Until homogenous suspension was achieved, APS aqueous solution ( $2 \mathrm{mmol}$ of APS in $10 \mathrm{~mL}$ of deionized water) was dropwise added to the suspension. The
TABLE 1: Composite absorbing material of CBP with different mass ratio.

\begin{tabular}{lc}
\hline Samples & Composition of samples \\
\hline $\mathrm{CBP}_{1}$ & $\mathrm{CNTs} / \mathrm{BaTiO}_{3}:$ PANI (mass ratio) $=4: 1$ \\
$\mathrm{CBP}_{2}$ & $\mathrm{CNTs}_{\mathrm{BaTiO}}:$ PANI (mass ratio) $=3: 2$ \\
$\mathrm{CBP}_{3}$ & $\mathrm{CNTs}_{3} / \mathrm{BaTiO}_{3}:$ PANI (mass ratio) $=2: 3$ \\
$\mathrm{CBP}_{4}$ & $\mathrm{CNTs} / \mathrm{BaTiO}_{3}:$ PANI (mass ratio) $=1: 4$ \\
\hline
\end{tabular}

polymerization process was applied in an ice bath for $24 \mathrm{~h}$ under stirring. The resulting precipitations were washed with deionized water and ethanol, followed by drying in an oven $\left(50^{\circ} \mathrm{C}\right)$ to obtain PANI.

$\mathrm{CNTs} / \mathrm{BaTiO}_{3} / \mathrm{PANI}$ (CBP) composites were obtained by using mechanical method as follows: different mass ratios of the as-prepared $\mathrm{CNT} / \mathrm{BaTiO}_{3}$ and PANI were loaded in a stainless jar and ball-milled by planetary ball mill (Retsch PM 100) at a rotation speed of $250 \mathrm{rpm}$ for $2 \mathrm{~h}$. The specific parameters were shown in Table 1.

2.5. Characterization. The morphology of samples was observed by scanning electron microcopy (FE-SEM; Hitachi S-4800), and the crystal structure of the prepared powders was analyzed with an X-ray diffractometer (Bruker AXS, D8Discover), using $\mathrm{Cu} \mathrm{K} \alpha$ radiation. Fourier transform infrared spectroscopy (FT-IR) was performed using a Nicolet 5700 FT-IR spectrometer (Thermo Electron Corp., USA) with $\mathrm{KBr}$ pellets. Conductivity was measured by four-point probe (SZT-2B). The composite samples used for electromagnetic measurements were prepared by loading the products in paraffin wax $(30 \mathrm{wt} \% \mathrm{CB}$ or CBP composites were mixed with wax). The powder-wax compound was then pressed into toroidally shaped samples $\left(\varphi_{\text {out }}=7 \mathrm{~mm}\right.$ and $\left.\varphi_{\text {inner }}=3 \mathrm{~mm}\right)$ for complex permittivity $\varepsilon\left(\varepsilon=\varepsilon^{\prime}-j \varepsilon^{\prime \prime}\right)$ and permeability $\mu$ $\left(\mu=\mu^{\prime}-j \mu^{\prime \prime}\right)$ measurements with a vector network analyzer (N5224A, Agilent) in the $2-18 \mathrm{GHz}$ range.

\section{Results and Discussion}

3.1. Morphology and Structure Analysis. Representative FESEM images of PANI, CNTs, and CB are shown in Figures 1(a)-1(c). The morphology of PANI is displayed in Figure 1(a) as short rod-like, and the average diameter is about $55.8 \mathrm{~nm}$ (Figure 1(d)). For the CNTs, seen in Figure 1(b), the nanotubes with smooth surface and diameter in the rage of $20-90 \mathrm{~nm}$ can be clearly observed (Figure 1(e)). The morphology of CB presented in Figure 1(c) indicates that the $\mathrm{BaTiO}_{3}$ is mounted onto the surface of the CNTs uniformly, and the surface is no longer smooth.

The morphology of $\mathrm{CBP}_{1}, \mathrm{CBP}_{2}, \mathrm{CBP}_{3}$, and $\mathrm{CBP}_{4}$ presented in Figures 2(a)-2(d) shows that homogeneous composites of CBP have formed. In attention, it can be seen that a 3D network structure has been built up between PANI and CB. Meanwhile, significant effect of synergic would form from the network, which could distinctly enhance the wave absorption.

The XRD patterns are shown in Figure 3. For pure PANI, the characteristic diffraction peaks at around $2 \theta=15.4^{\circ}, 20.4^{\circ}$, 


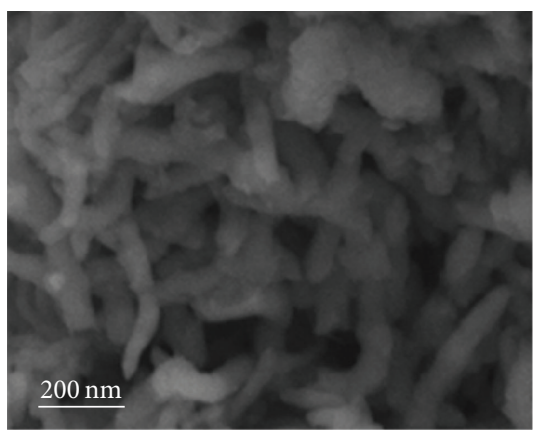

(a)

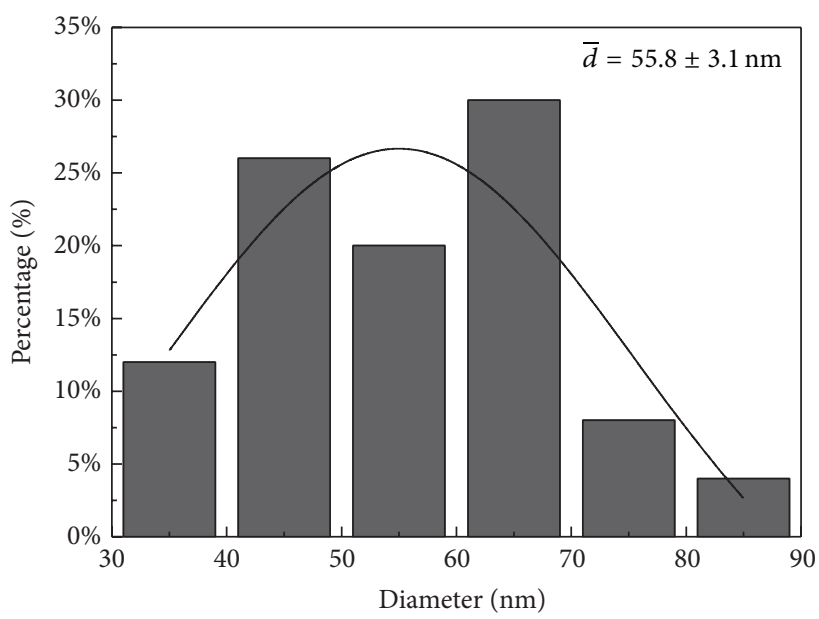

(d)

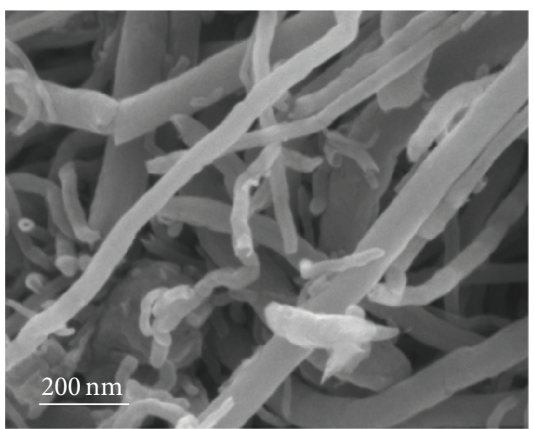

(b)

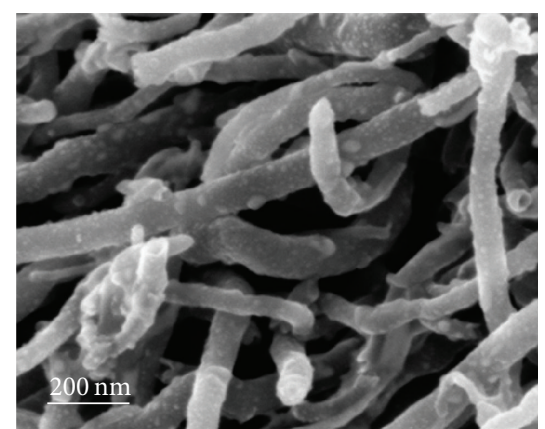

(c)

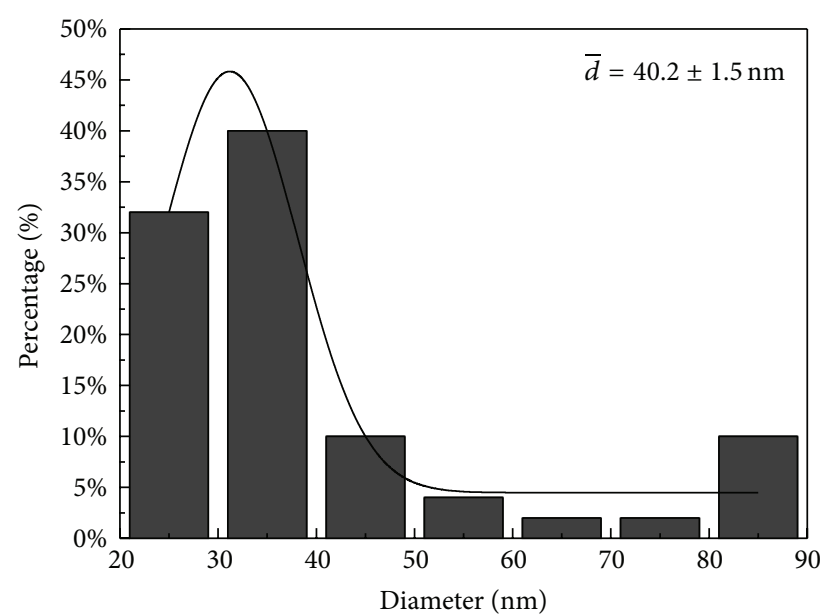

(e)

Figure 1: FE-SEM images of (a) PANI, (b) CNTs, and (c) CB. Diameter of (d) PANI and (e) CNTs.

and $25.1^{\circ}$ are observed. Among them, $2 \theta=15.4^{\circ}$ and $20.4^{\circ}$ are attributed to the periodicity both perpendicular and parallel to the polymer chain, respectively, and the characteristic peak at $2 \theta=25.1^{\circ}$ is caused by the face-to-face interchain stacking distance between phenyl rings [15-17]. For CB, the diffraction peak at $2 \theta=26.2^{\circ}$ originates from the CNTs (002) [18], and the week diffraction peak may be due to wrapped $\mathrm{BaTiO}_{3}$. The diffraction peaks at $2 \theta=22.2^{\circ}, 31.6^{\circ}, 39.0^{\circ}, 45.3^{\circ}, 51.0^{\circ}$, $56.2^{\circ}$, and $65.8^{\circ}$ can be indexed to the (100), (110), (111), (200), (210), (211), and (220) planes of $\mathrm{BaTiO}_{3}$ (JCPDS card number 31-0174) [19]. Meanwhile, it can also observed from the CBP, with decreasing $\mathrm{CB}$ content in composite, the decrease in the intensity peaks of $\mathrm{BaTiO}_{3}$ along with the increase in the intensity peak of PANI at $2 \theta=25.1^{\circ}$, which overlapped with the (002) peak of CNTs. Additionally, characteristic diffraction peaks of $\mathrm{BaTiO}_{3}$ keep consistent in the CBP which indicate that the crystal phase structure of $\mathrm{BaTiO}_{3}$ was not changed.

Functional groups of samples are also detected by FTIR measurement, as shown in Figure 4. For PANI, the characteristic peaks at $1560 \mathrm{~cm}^{-1}$ and $1475 \mathrm{~cm}^{-1}$ are attributed to the $\mathrm{C}=\mathrm{C}$ stretching vibration of the quinoid $(\mathrm{Q})$ ring and the benzenoid $(\mathrm{N})$ ring, respectively, indicating the presence of the emeraldine salt of PANI. The peak at $1300 \mathrm{~cm}^{-1}$ is due to the $\mathrm{C}-\mathrm{N}$ stretching vibration in PANI. The peak at $1241 \mathrm{~cm}^{-1}$ is assigned to the stretching vibration of the $\mathrm{CN}^{\circ+}$ in the polaron structure of PANI [20-22]. The characteristic peak at $1106 \mathrm{~cm}^{-1}$ can be attributed to the stretching of $\mathrm{C}=\mathrm{N}(\mathrm{N}=\mathrm{Q}=\mathrm{N})$. For the $\mathrm{CB}$, the absorption bands centered at $518 \mathrm{~cm}^{-1}$ and $430 \mathrm{~cm}^{-1}$ are due to Ti-O stretching vibrations and characteristic of $\mathrm{BaTiO}_{3}[23,24]$. For the CBP, the peaks of $\mathrm{CB}$ almost cannot be detected due to the strong absorption peaks of PANI. Meanwhile, neither blueshift nor redshift happened in the spectrum of CBP (dash lines are shown in Figure 4), indicating that the structure of CB and PANI was not changed, which is consistent with the results of XRD.

The conductivity property of samples was measured, as shown in Figure 5. For CNTs (treated by $\mathrm{HNO}_{3}$ ), it can be seen that the electrical conductivity is about $1.1202 \mathrm{~S} / \mathrm{cm}$. Also, it is clear that the conductivity of CBP increased gradually with increasing the content of PANI in the system. Particularly, the conductivity of pure PANI reached $3.7425 \mathrm{~S} / \mathrm{cm}$. Here it should be pointed out that conductivity of these samples falls in the range from $10^{-2} \mathrm{~S} / \mathrm{cm}$ to $10^{1} \mathrm{~S} / \mathrm{cm}$ desired for exhibiting good microwave absorb responses [25]. Therefore, conductivity of CBP may be beneficial to the enhancement of microwave absorption.

3.2. Electromagnetic Wave Absorption Properties of $C B P$ Composites. It is believed that the complex permittivity and permeability of the absorber determine the microwave 


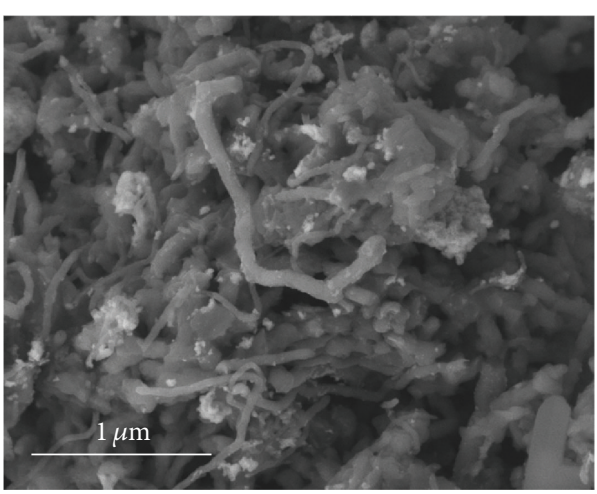

(a)

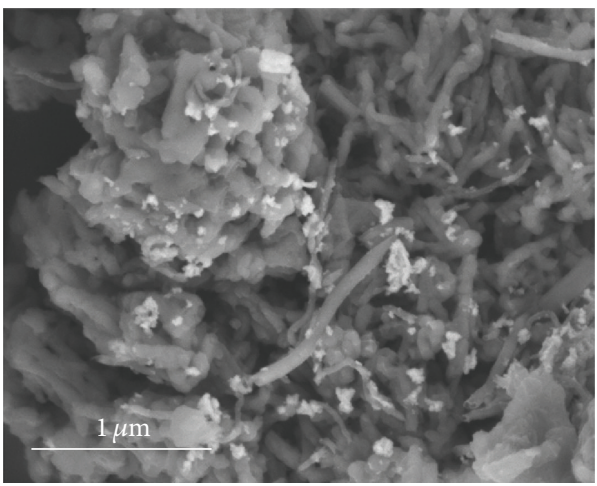

(c)

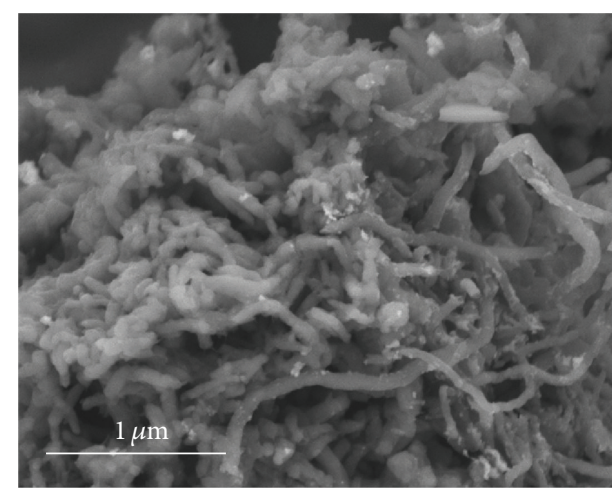

(b)

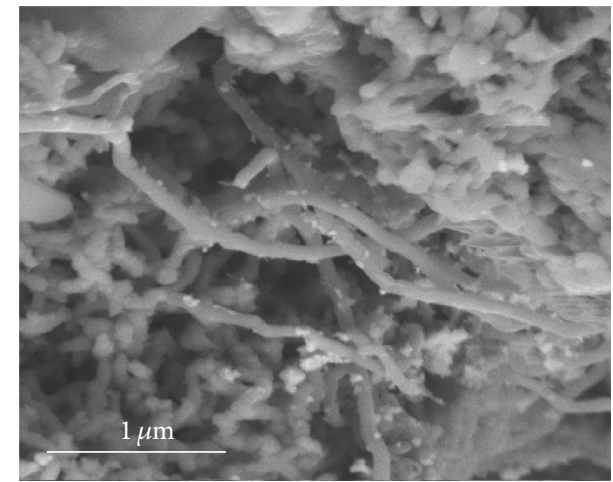

(d)

Figure 2: FE-SEM images of (a) $\mathrm{CBP}_{1}$, (b) $\mathrm{CBP}_{2}$, (c) $\mathrm{CBP}_{3}$, and (d) $\mathrm{CBP}_{4}$.

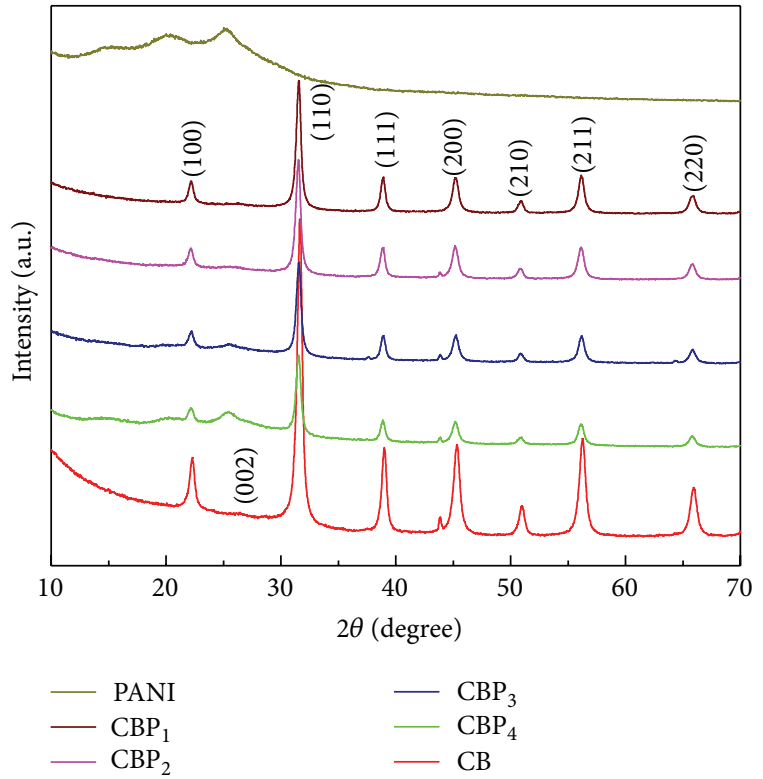

Figure 3: XRD pattern of PANI, $\mathrm{CBP}_{1}, \mathrm{CBP}_{2}, \mathrm{CBP}_{3}, \mathrm{CBP}_{4}$, and $\mathrm{CB}$.

absorption properties. In order to evaluate the microwave absorption properties of CB and CBP, the complex permittivity and permeability of the composites were measured in frequency range of $2-18 \mathrm{GHz}$. As shown in Figure 6(a), $\varepsilon^{\prime}$

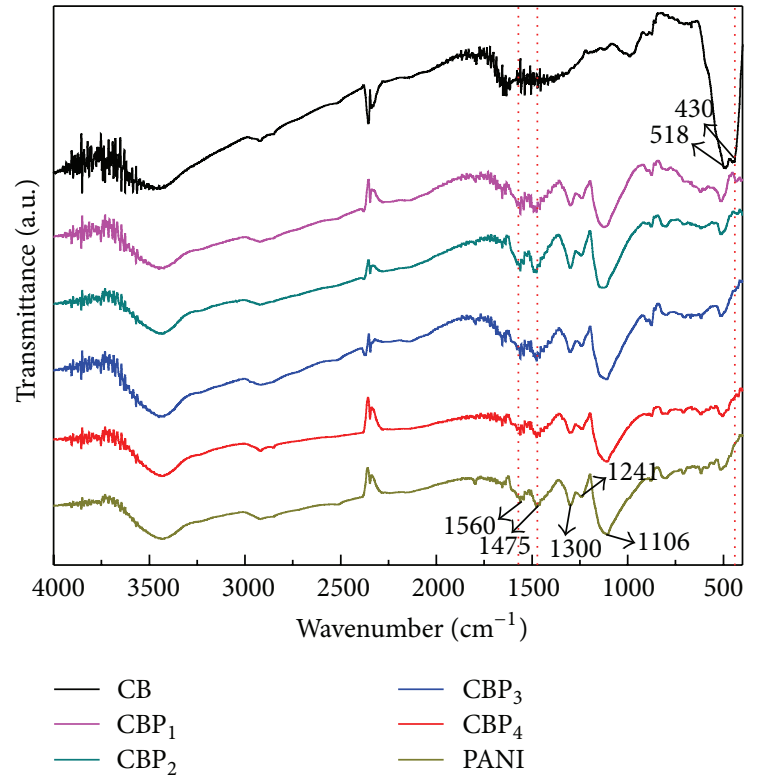

FIgURE 4: FT-IR spectra of PANI, $\mathrm{CBP}_{1}, \mathrm{CBP}_{2}, \mathrm{CBP}_{3}, \mathrm{CBP}_{4}$, and CB.

of CBP slight increases with increasing of PANI content. It can be ascribed to the "geometrical effect" of formed $3 \mathrm{D}$ conductive network structures and the strong interfacial polarization effects enhanced between conducting PANI and 


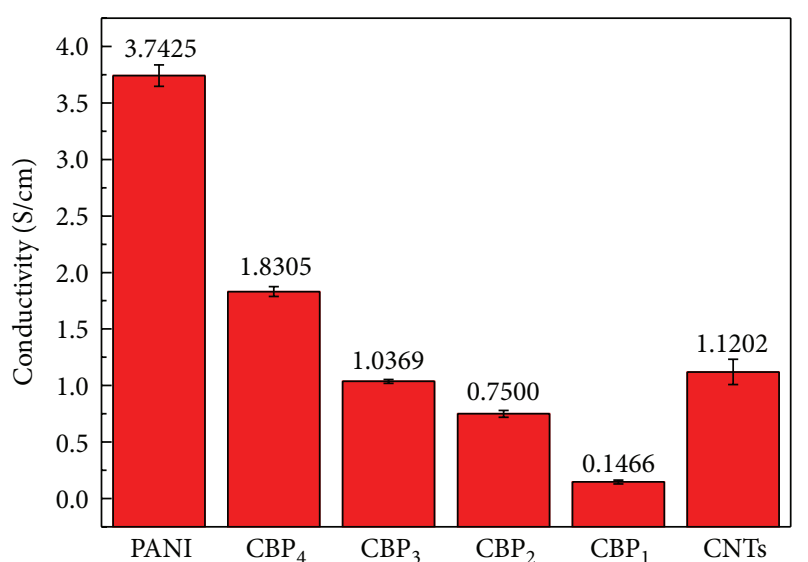

FIGURE 5: Electrical conductivity of PANI, $\mathrm{CBP}_{4}, \mathrm{CBP}_{3}, \mathrm{CBP}_{2}, \mathrm{CBP}_{1}$, and CNTs.

$\mathrm{CB}$, leading to improvement of dielectric constant. As shown in Figure 6(b), for all samples, the variation tendency of the imaginary parts $\varepsilon^{\prime \prime}$ of the complex permeability was similar to the real parts $\varepsilon^{\prime}$. Particularly, several relaxation peaks on $\varepsilon^{\prime \prime}$ curves were observed. It can be attributed to typical characteristics of nonlinear resonant behaviors [26]. As mentioned above, the value of complex permittivity can be improved effectively with the appropriate content of PANI.

The complex permeability spectra of the CB and CBP composites are presented in Figure 7. It is obviously exhibited that the real part $\mu^{\prime}$ and imaginary part $\mu^{\prime \prime}$ of samples remain almost constant in the whole frequency range with the value being about 1 and 0 , respectively. It can be concluded that the main contribution to the microwave absorption of samples results from the dielectric loss rather than the magnetic loss.

To further study the microwave absorption properties, the reflection loss (RL) properties of samples were calculated according to transmission line theory as follows:

$$
\mathrm{RL}=20 \log \left|\frac{Z_{\text {in }}-1}{Z_{\text {in }}+1}\right| .
$$

The normalized input impedance $\left(Z_{\text {in }}\right)$ is given by the formula:

$$
Z_{\text {in }}=\sqrt{\frac{\mu_{r}}{\varepsilon_{r}}} \tanh \left[j\left(\frac{2 \pi f d}{c}\right) \sqrt{\mu_{r} \varepsilon_{r}}\right],
$$

where $f$ is the microwave frequency in $\mathrm{Hz}, d$ is the thickness of the absorber in $\mathrm{m}, c$ is the velocity of light in free space in $\mathrm{m} / \mathrm{s}$, and $\varepsilon_{r}$ and $\mu_{r}$ are the complex permittivity and permeability, respectively. Based on the electromagnetic parameters (the complex values of permittivity and permeability), the $\mathrm{RL}$ can be calculated for the given frequency with various thicknesses according to (1) and (2).

The calculated reflection loss curves of composites in the range of $2-18 \mathrm{GHz}$ are shown in Figure 8 . Reflection loss of $\mathrm{CB}$ and CBP samples at a thickness of $4 \mathrm{~mm}$ is shown in Figure 8(a); it can be observed that the sample of $\mathrm{CBP}_{3}$ exhibits high-performance microwave absorption compared with $\mathrm{CB}$ and others. It shows that the minimum RL value

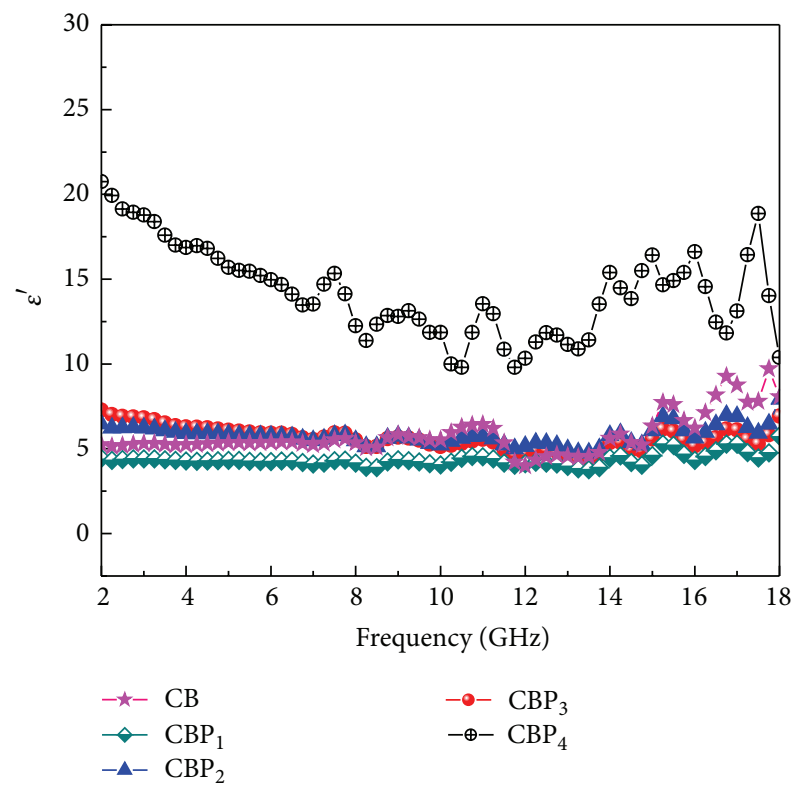

(a)

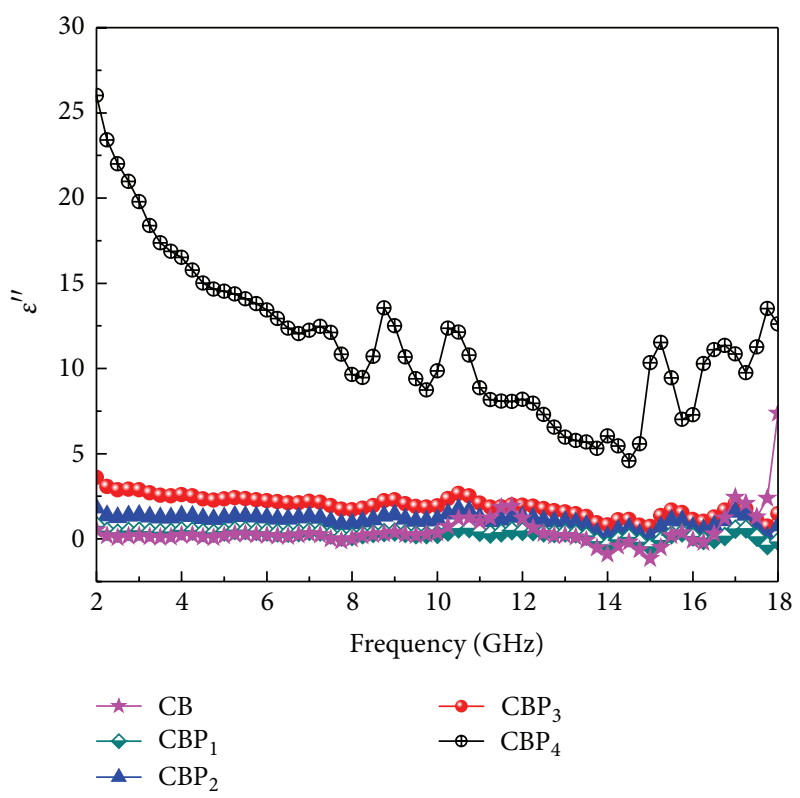

(b)

FIGURE 6: Complex permittivity of samples: (a) real part and (b) imaginary part.

is up to $-30.9 \mathrm{~dB}$ at $8 \mathrm{GHz}$ and bandwidth corresponding to the reflection loss lower than $-10 \mathrm{~dB}$ is from $7.5 \mathrm{GHz}$ to 10.2 GHz. The excellent microwave absorption of the $\mathrm{CBP}_{3}$ can be accounted by the fact that the prominent interfacial polarization and synergistic action are formed due to the interaction between conductive network of PANI and electric field. Furthermore, impedance matching has been improved with the appropriate content of PANI. Nevertheless, the microwave absorption of $\mathrm{CBP}_{4}$ is sharply deteriorated with the PANI content of composites further increasing. The reason is that a high value of permittivity and conductivity 


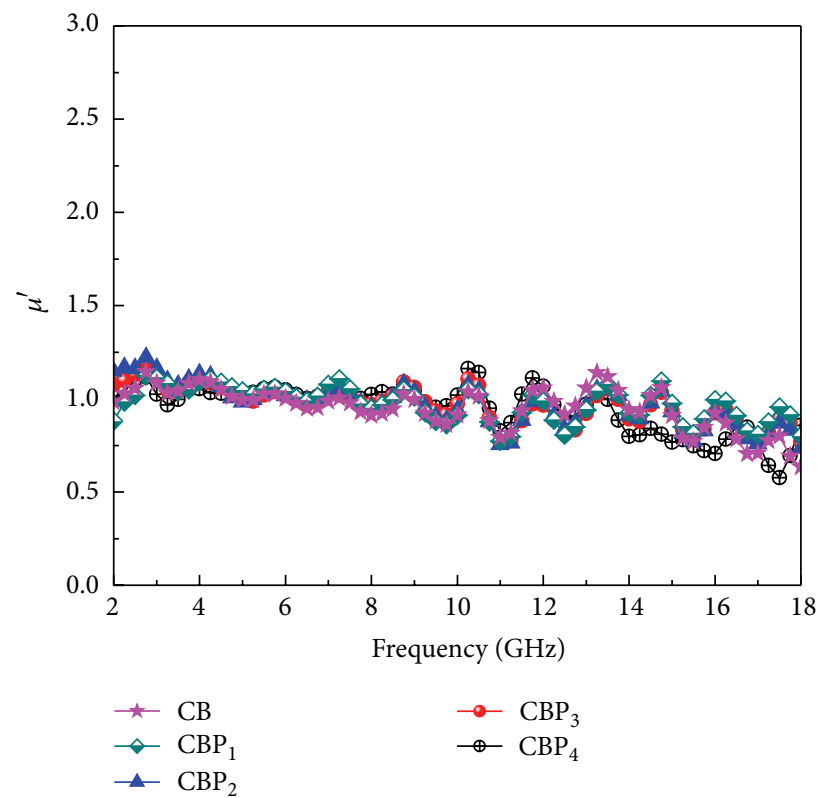

(a)

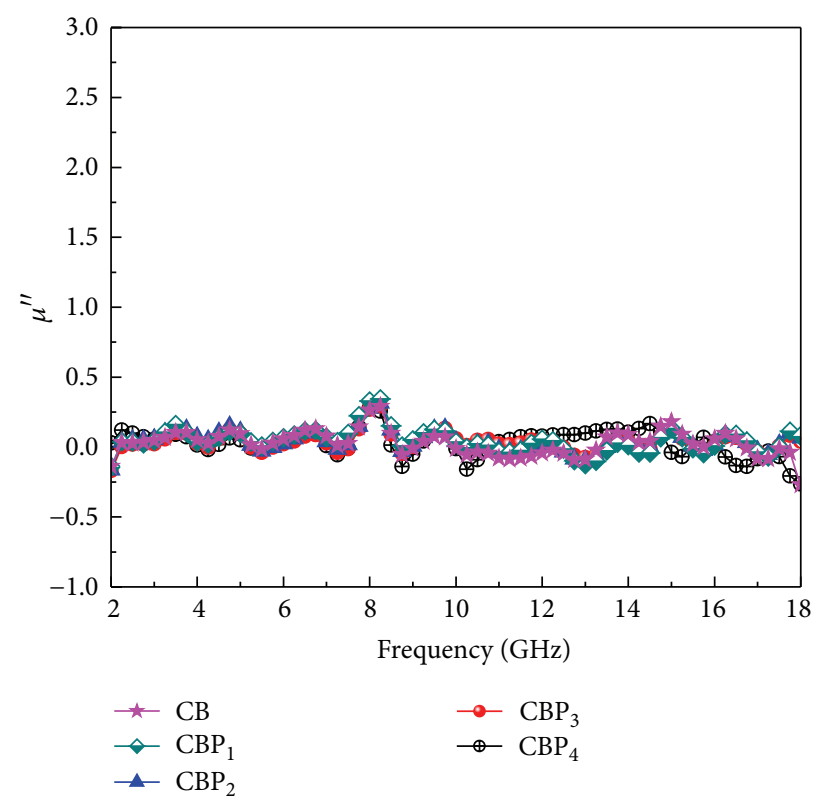

(b)

FIGURE 7: Complex permeability of samples: (a) real part and (b) imaginary part.

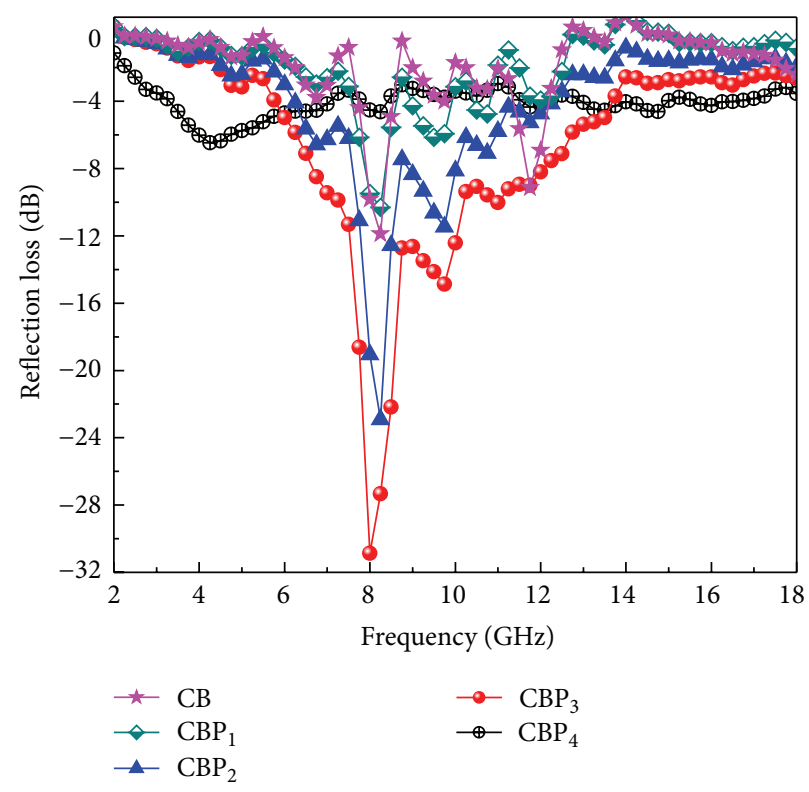

(a)

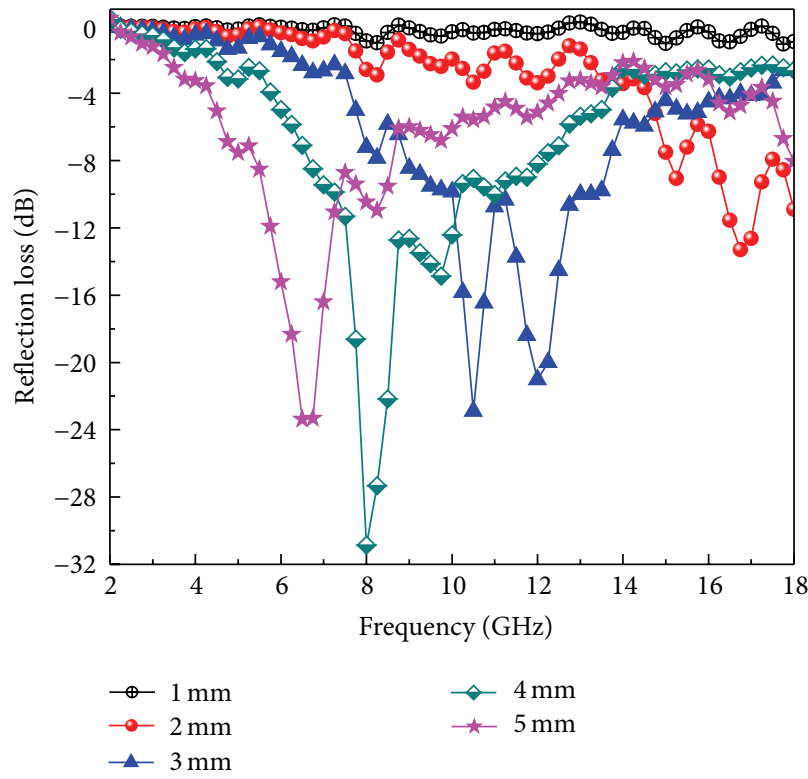

(b)

Figure 8: (a) Reflection loss of samples with the thickness of $4 \mathrm{~mm}$ and (b) reflection loss of $\mathrm{CBP}_{3}$ with different thickness.

will result in strong reflection and poor impedance matching. The reflection loss curves of $\mathrm{CBP}_{3}$ with different thicknesses are shown in Figure 8(b). It is clear that the minimum reflection loss peak shifted to the low frequency with the increasing thickness, which is corresponding to the theory of quarter-wave principle $[27,28]$. Based on above analysis, it concludes that the high-performance microwave absorption of CBP can be improved significantly by tunable components.
The main microwave absorption mechanism of CBP is proposed in Figure 9. It is well known that the microwave energy attenuation by CNTs and $\mathrm{CB}$ is relying on electronic polarization and interfacial polarization. Therefore, microwave absorption of composites can be enhanced through dielectric polarization relaxation for the CNTs decorated with $\mathrm{BaTiO}_{3}$ nanoparticles. In addition, it is also noted that network formed between PANI and CB not only 


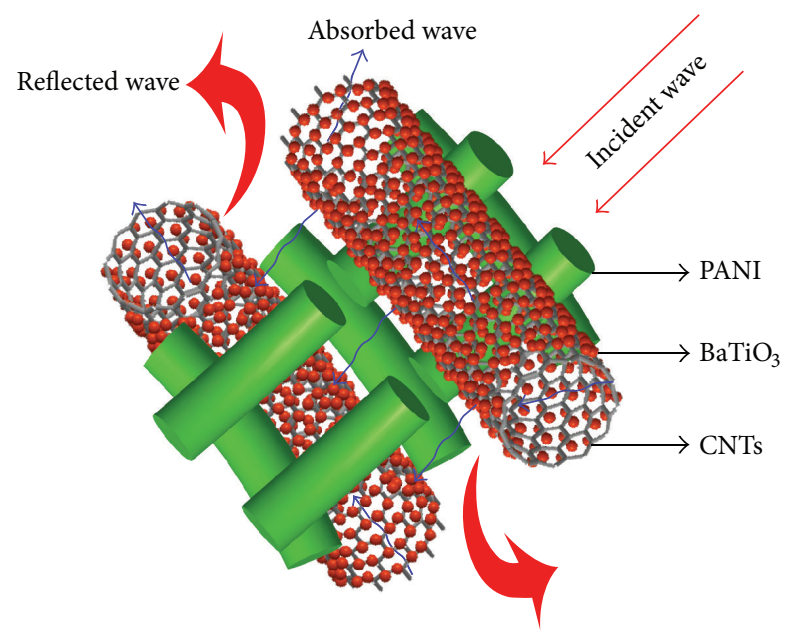

FIgURE 9: Electromagnetic wave absorbing mechanism of CBP composite.

reinforcing synergistic action but also strengthening interfacial polarization. Furthermore, the impedance matching properties of materials have been improved significantly by PANI. Thus, compared with $\mathrm{CB}$, the microwave absorption of CBP is improved significantly.

\section{Conclusions}

Barium titanate/carbon nanotubes incorporated polyaniline composites with 3D conductive network structure have been successfully prepared by an easy and flexible method, which exhibit excellent microwave absorbing properties. The good dielectric properties and high impedance matching of CBP composites are due to presence of $\mathrm{CB}$ and PANI, as well as the synergistic effect and geometrical effect due to the microstructure which collectively contributes towards the outstanding performance. The CBP with the mass ratio of $\mathrm{CB}: \mathrm{PANI}=2: 3\left(\mathrm{CBP}_{3}\right)$ shows the best microwave absorption properties. The maximum reflection loss of $\mathrm{CBP}_{3}$ is $-30.9 \mathrm{~dB}$ at $8 \mathrm{GHz}$ with a $4 \mathrm{~mm}$ thick sample layer and filler loading of $30 \mathrm{wt} \%$ paraffin wax, and the bandwidth with a reflection loss less than $-10 \mathrm{~dB}$ covers wide frequency range from 7.5 to $10.2 \mathrm{GHz}$. Therefore, it is reasonable to believe their potential for making futuristic microwave absorbers.

\section{Competing Interests}

The authors declare no competing financial interests.

\section{Acknowledgments}

This work issupported by National Natural Science Foundation of China (no. 51503183); Zhejiang Provincial Natural Science Foundation of China (no. LQ14E030010); Science Foundation of Zhejiang Sci-Tech University (ZSTU) (nos. 13012147-Y and 13012062-Y); Zhejiang Top Priority Discipline of Textile Science and Engineering, National Engineering Lab for Textile Fiber Materials and Processing Technology (Zhejiang) (nos. 2013YBZX04 and 2014CLXK10). The authors also thank Professor Yida Deng of Shanghai Jiangtong University for the help of microwave absorption measurements.

\section{References}

[1] F. F. Xu, L. Ma, M. Y. Gan et al., "Preparation and characterization of chiral polyaniline/barium hexaferrite composite with enhanced microwave absorbing properties," Journal of Alloys and Compounds, vol. 593, pp. 24-29, 2014.

[2] Q. Q. He, T. T. Yuan, X. Zhang et al., "Electromagnetic field absorbing polypropylene nanocomposites with tuned permittivity and permeability by nanoiron and carbon nanotubes," Journal of Physical Chemistry C, vol. 118, no. 42, pp. 2478424796, 2014.

[3] J. Zhu, S. Wei, N. Haldolaarachchige, D. P. Young, and Z. Guo, "Electromagnetic field shielding polyurethane nanocomposites reinforced with core-shell Fe-silica nanoparticles," Journal of Physical Chemistry C, vol. 115, no. 31, pp. 15304-15310, 2011.

[4] Y. Qing, Y. Mu, Y. Zhou, F. Luo, D. Zhu, and W. Zhou, "Multiwalled carbon nanotubes- $\mathrm{BaTiO}_{3} /$ silica composites with high complex permittivity and improved electromagnetic interference shielding at elevated temperature," Journal of the European Ceramic Society, vol. 34, no. 10, pp. 2229-2237, 2014.

[5] Z. Zheng, B. Xu, L. Huang, L. He, and X. Ni, "Novel composite of Co/carbon nanotubes: synthesis, magnetism and microwave absorption properties," Solid State Sciences, vol. 10, no. 3, pp. 316-320, 2008.

[6] H. F. Li, J. Wang, Y. H. Huang et al., "Microwave absorption properties of carbon nanotubes and terapod-shaped $\mathrm{ZnO}$ nanostructures," Materials Science and Engineering B, vol. 175, no. 1, pp. 81-85, 2010.

[7] Y.-F. Zhu, Y.-Q. Fu, T. Natsuki, and Q.-Q. Ni, "Fabrication and microwave absorption properties of $\mathrm{BaTiO}_{3}$ nanotube/polyaniline hybrid nanomaterials," Polymer Composites, vol. 34, no. 2, pp. 265-273, 2013.

[8] Z. Guo, S.-E. Lee, H. Kim et al., "Fabrication, characterization and microwave properties of polyurethane nanocomposites reinforced with iron oxide and barium titanate nanoparticles," Acta Materialia, vol. 57, no. 1, pp. 267-277, 2009.

[9] X. Huang, Z. R. Chen, L. F. Tong, M. N. Feng, Z. J. Pu, and X. B. Liu, "Preparation and microwave absorption properties of $\mathrm{BaTiO}_{3} @ \mathrm{MWCNTs}$ core/shell heterostructure," Materials Letters, vol. 111, pp. 24-27, 2013.

[10] Y.-F. Zhu, Q.-Q. Ni, and Y.-Q. Fu, "One-dimensional barium titanate coated multi-walled carbon nanotube heterostructures: synthesis and electromagnetic absorption properties," RSC Advances, vol. 5, no. 5, pp. 3748-3756, 2015.

[11] Q. S. Wang, Z. Y. Lei, Y. J. Chen et al., "Branched polyaniline/molybdenum oxide organic/inorganic heteronanostructures: synthesis and electromagnetic absorption properties," Journal of Materials Chemistry A, vol. 1, no. 38, pp. 11795-11801, 2013.

[12] H. B. Gu, J. Guo, X. R. Yan et al., "Electrical transport and magnetoresistance in advanced polyaniline nanostructures and nanocomposites," Polymer, vol. 55, no. 17, pp. 4405-4419, 2014.

[13] J. Guo, J. Long, D. Ding et al., "Significantly enhanced mechanical and electrical properties of epoxy nanocomposites reinforced with low loading of polyaniline nanoparticles," RSC Advances, vol. 6, no. 25, pp. 21187-21192, 2016. 
[14] C. L. Yang, H. G. Wei, L. Guan et al., "Polymer nanocomposites for energy storage, energy saving, and anticorrosion," Journal of Materials Chemistry A, vol. 3, no. 29, pp. 14929-14941, 2015.

[15] K. Lee, S. Cho, H. P. Sung, A. J. Heeger, C.-W. Lee, and S.-H. Lee, "Metallic transport in polyaniline," Nature, vol. 441, no. 1, pp. 65-68, 2006.

[16] X. Li, M. Zhou, H. Xu, G. Wang, and Z. Wang, "Synthesis and electrochemical performances of a novel two-dimensional nanocomposite: polyaniline-coated laponite nanosheets," Journal of Materials Science, vol. 49, no. 19, pp. 6830-6837, 2014.

[17] M. X. Wan, J. C. Li, and S. Z. Li, "Microtubules of polyaniline as new microwave absorbent materials," Polymers for Advanced Technologies, vol. 12, no. 11-12, pp. 651-657, 2001.

[18] G. Gao, D. Guo, C. Wang, and H. Li, "Electrocrystallized Ag nanoparticle on functional multi-walled carbon nanotube surfaces for hydrazine oxidation," Electrochemistry Communications, vol. 9, no. 7, pp. 1582-1586, 2007.

[19] X. D. Chen, G. Q. Wang, Y. P. Duan, and S. H. Liu, "Electromagnetic characteristics of barium titanate/epoxide resin composites in X and Ku bands," Journal of Alloys and Compounds, vol. 453, no. 1-2, pp. 433-436, 2008.

[20] D. D. Potphode, P. Sivaraman, S. P. Mishra, and M. Patri, "Polyaniline/partially exfoliated multi-walled carbon nanotubes based nanocomposites for supercapacitors," Electrochimica Acta, vol. 155, pp. 402-410, 2015.

[21] H. Fan, H. Wang, N. Zhao, X. Zhang, and J. Xu, "Hierarchical nanocomposite of polyaniline nanorods grown on the surface of carbon nanotubes for high-performance supercapacitor electrode," Journal of Materials Chemistry, vol. 22, no. 6, pp. 27742780, 2012.

[22] L. Li, Z.-Y. Qin, X. Liang et al., "Facile fabrication of uniform core-shell structured carbon nanotube-polyaniline nanocomposites," The Journal of Physical Chemistry C, vol. 113, no. 14, pp. 5502-5507, 2009.

[23] H. C. Pant, M. K. Patra, A. Verma, S. R. Vadera, and N. Kumar, "Study of the dielectric properties of barium titanate-polymer composites," Acta Materialia, vol. 54, no. 12, pp. 3163-3169, 2006.

[24] H. Yan, Z. J. Liao, X. Zhu et al., "Synthesis and electrorheological properties of polyaniline-coated barium titanate composite particles," Journal of Applied Polymer Science, vol. 107, no. 3, pp. 1960-1966, 2008.

[25] Q.-Q. Ni, Y.-F. Zhu, L.-J. Yu, and Y.-Q. Fu, "Onedimensional carbon nanotube@barium titanate@polyaniline multiheterostructures for microwave absorbing application," Nanoscale Research Letters, vol. 10, no. 1, p. 174, 2015.

[26] B. Zhao, G. Shao, B. Fan, W. Guo, Y. Chen, and R. Zhang, "Preparation of $\mathrm{SnO}_{2}$-coated Ni microsphere composites with controlled microwave absorption properties," Applied Surface Science, vol. 332, pp. 112-120, 2015.

[27] C. Wang, X. Han, X. Zhang et al., "Controlled synthesis and morphology-dependent electromagnetic properties of hierarchical cobalt assemblies," Journal of Physical Chemistry C, vol. 114, no. 35, pp. 14826-14830, 2010.

[28] Y. C. Qing, D. D. Min, Y. Y. Zhou, F. Luo, and W. C. Zhou, "Graphene nanosheet- and flake carbonyl iron particlefilled epoxy-silicone composites as thin-thickness and widebandwidth microwave absorber," Carbon, vol. 86, pp. 98-107, 2015. 

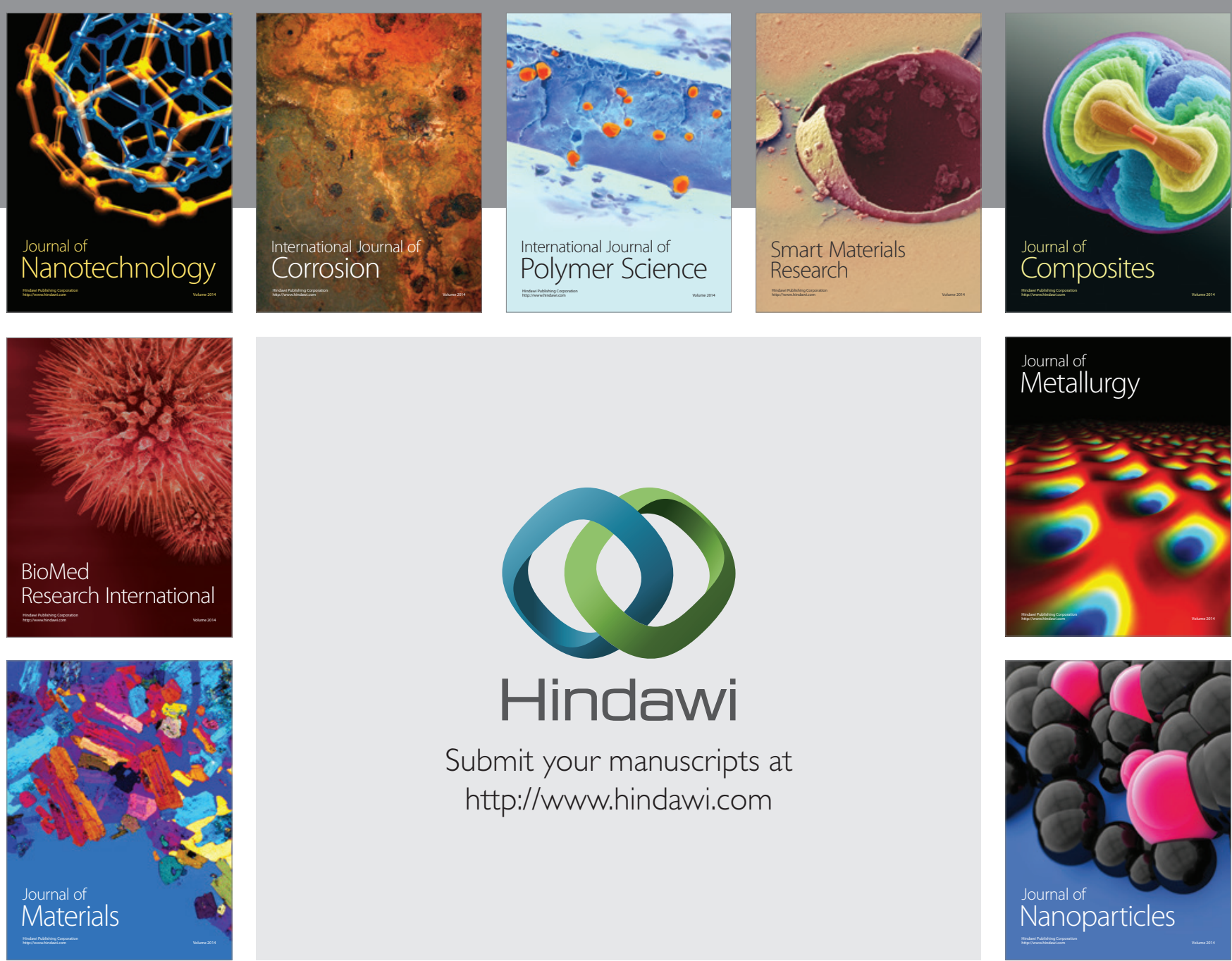

\section{Hindawi}

Submit your manuscripts at

http://www.hindawi.com

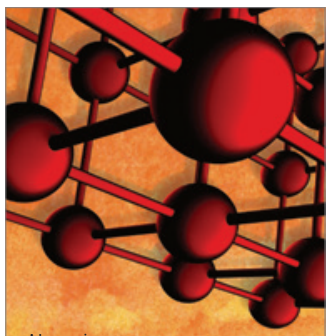

Materials Science and Engineering
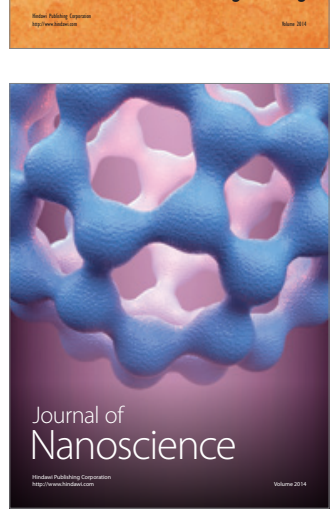
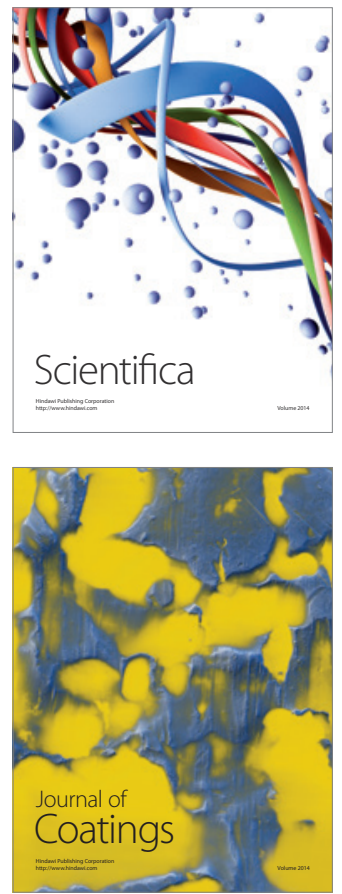
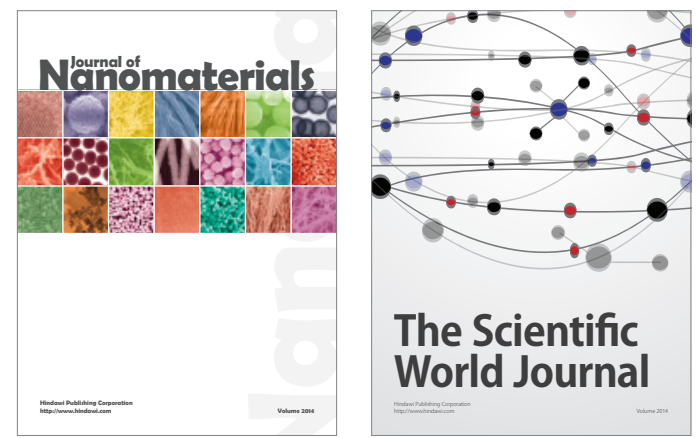

The Scientific World Journal
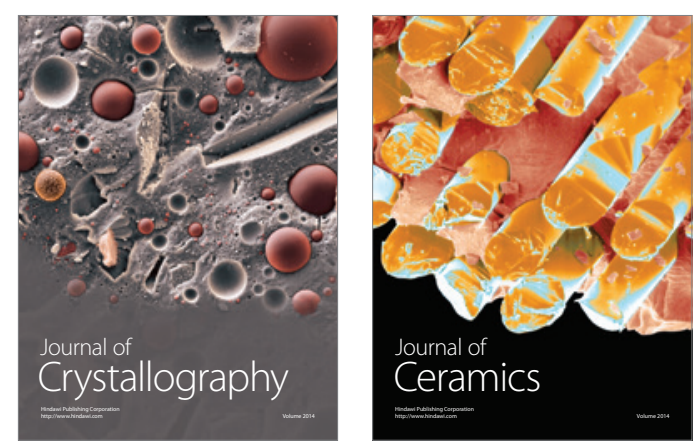
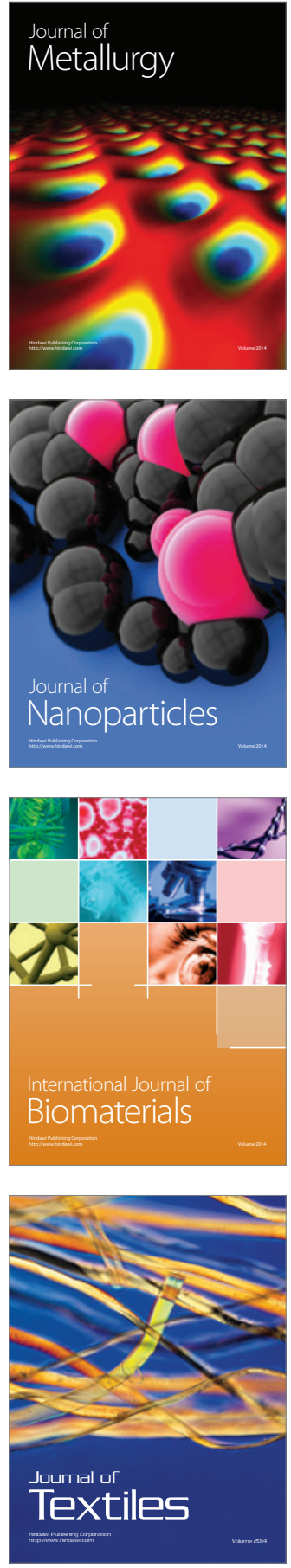